\title{
Limits of interval orders and semiorders
}

\author{
SVANTE JANSON
}

\begin{abstract}
We study poset limits given by sequences of finite interval orders or, as a special case, finite semiorders. In the interval order case, we show that every such limit can be represented by a probability measure on the space of closed subintervals of $[0,1]$, and we define a subset of such measures that yield a unique representation. In the semiorder case, we similarly find unique representations by a class of distribution functions.
\end{abstract}

\section{Introduction and main results}

The theory of graph limits was founded by by Lovász and Szegedy [13] and Borgs, Chayes, Lovász, Sós and Vesztergombi [3, 4], and further developed in a series of papers by these and other authors. An analogous theory for poset limits was initiated by Brightwell and Georgiou [5] and further developed by Janson [11]. The purpose of the present paper is to study the special cases of limits of interval orders and semiorders. (Cf. the related study of interval graph limits in [7].)

Definitions of these classes of posets and our main results are given in Sections 4 and 5, after some preliminaries. We show there that every interval order limit can be represented by a probability measure on the space $\{[a, b]: 0 \leq a \leq b \leq 1\}$ of closed subintervals of $[0,1]$, and that every semiorder limit can be represented by a weakly increasing function $g:[0,1] \rightarrow[0,1]$ such that $g(x) \geq x$. Moreover, unlike most previously studied cases of similar representations of graph limits or poset limits, in these two cases we find explicit classes of such measures and functions that yield unique representations. For semiorders, this leads to necessary and sufficient conditions for a sequence of semiorders to converge to a semiorder limit; these conditions use the distributions of the numbers of predecessors or successors of points in the semiorders.

In Section 6 we discuss the connections to graph limits, including some open problems.

arXiv: 1104.1264 


\section{Preliminaries}

We assume that the reader is familiar with the theory of graph limits and the poset version of it in [11]. We use the same notations as there (see also the graph case in [8]); for convenience, we repeat the main definitions.

All posets are assumed to be non-empty. They are usually finite, but we will sometimes use infinite posets as well. If $(P,<)$ is a poset, we call $P$ its ground set. For simplicity, we use the same notation for a poset and its ground set when there is no danger of confusion. We let $\mathcal{P}$ denote the set of unlabelled finite posets.

We may regard a poset $(P,<)$ as a digraph, with vertex set $P$ and a directed edge $i \rightarrow j$ if and only if $i<j$ for all $i, j \in P$. (But note that not every digraph is a poset.)

The functional $t(Q, P)$ is defined for finite posets $P$ and $Q$ as the proportion of all maps $\varphi: Q \rightarrow P$ that are poset homomorphisms, i.e., such that $x<_{Q} y \Longrightarrow \varphi(x)<_{P} \varphi(y)$. We similarly also define $t_{\text {inj }}(Q, P)$ as the proportion of all injective maps $Q \rightarrow P$ that are poset homomorphisms and $t_{\text {ind }}(Q, P)$ as the proportion of all injective maps $\varphi: Q \rightarrow P$ such that $x<_{Q} y \Longleftrightarrow \varphi(x)<_{P} \varphi(y)$ (i.e., $\varphi$ is an isomorphism onto an induced subposet of $P$ ); equivalently, $t_{\text {ind }}(Q, P)$ is the probability that a random labelled induced subposet of $|Q|$ points in $P$ is isomorphic to $Q$ (for any fixed labelling of $Q$ ). (If $|Q|>|P|$, we define $t_{\text {inj }}(Q, P)=t_{\text {ind }}(Q, P)=0$.)

We say that a sequence $\left(P_{n}\right)$ of finite posets with $\left|P_{n}\right| \rightarrow \infty$ converges, if $t\left(Q, P_{n}\right)$ converges for every finite poset $Q$. (All unspecified limits in this paper are as $n \rightarrow \infty$.) It is easy to see that this is equivalent to convergence of $t_{\text {inj }}\left(Q, P_{n}\right)$ for every $Q$, or of $t_{\text {ind }}\left(Q, P_{n}\right)$ for every $Q$.

The (discrete) space $\mathcal{P}$ of finite posets can be embedded as an open dense subspace of a compact metric space $\overline{\mathcal{P}}$, such that a sequence $P_{n}$ with $\left|P_{n}\right| \rightarrow \infty$ converges in the sense just given if and only if it converges in the metric space $\overline{\mathcal{P}}$. The space $\mathcal{P}_{\infty}:=\overline{\mathcal{P}} \backslash \mathcal{P}$ is the space of poset limits.

For each poset $Q$, the functionals $t(Q, \cdot), t_{\mathrm{inj}}(Q, \cdot)$ and $t_{\text {ind }}(Q, \cdot)$ extend to continuous functionals on $\overline{\mathcal{P}}$. A poset limit $\Pi$ is uniquely determined by the sequence of numbers $\{t(Q, \Pi)\}_{Q \in \mathcal{P}}$, and also by $\left\{t_{\text {ind }}(Q, \Pi)\right\}_{Q \in \mathcal{P}}$.

An ordered probability space $(\mathcal{S}, \mathcal{F}, \mu, \prec)$ is a probability space $(\mathcal{S}, \mathcal{F}, \mu)$ equipped with a partial order $\prec$ such that $\{(x, y): x \prec y\}$ is a measurable subset of $\mathcal{S} \times \mathcal{S}$.

A (poset) kernel on an ordered probability space $(\mathcal{S}, \mathcal{F}, \mu, \prec)$ is a measurable function $W: \mathcal{S} \times \mathcal{S} \rightarrow[0,1]$ such that, for $x, y, z \in \mathcal{S}$,

$$
\begin{aligned}
W(x, y)>0 & \Longrightarrow x \prec y, \\
W(x, y)>0 \text { and } W(y, z)>0 & \Longrightarrow W(x, z)=1 .
\end{aligned}
$$


When convenient, we may omit parts of the notation that are clear from the context and say, e.g., that $\mathcal{S}$ or $(\mathcal{S}, \mu)$ is a probability space or an ordered probability space.

For $n \in \mathbb{N}:=\{1,2, \ldots\}$, let $[n]:=\{1, \ldots, n\}$, and let $[\infty]:=\mathbb{N}$. Thus $[n]$ is a set of cardinality $n$ for all $n \in \mathbb{N} \cup\{\infty\}$.

Given a kernel $W$ on an ordered probability space $(\mathcal{S}, \mathcal{F}, \mu, \prec)$, we define for every $n \in \mathbb{N} \cup\{\infty\}$ a random poset $P(n, W)$ of cardinality $n$ by taking a sequence $\left(X_{i}\right)_{i=1}^{\infty}$ of i.i.d. points in $\mathcal{S}$ with distribution $\mu$; given $\left(X_{i}\right)$, we then define $P(n, W)$ to be $[n]$ with the random partial order $\prec^{*}$ such that $i \prec^{*} j$ with probability $W\left(X_{i}, X_{j}\right)$, with (conditionally) independent choices for different pairs $(i, j)$. (A convenient construction is to take auxiliary independent random variables $\xi_{i j} \sim U(0,1), i, j \in \mathbb{N}$, and then define $i \prec{ }^{*} j$ if and only if $\xi_{i j}<W\left(X_{i}, X_{j}\right)$.) We also use the notation $P(n, W, \mu)$ or $P(n, \mu)$ when we wish to emphasize the dependence on $\mu$.

Example 2.1. In this paper we are mainly interested in the case when $W(x, y)=\mathbf{1}[x \prec y]$ on some ordered probability space $(\mathcal{S}, \mu)$. (We use $\mathbf{1}[\mathcal{E}]$ to denote the indicator function of the event $\mathcal{E}$, which is 1 if $\mathcal{E}$ occurs and 0 otherwise.) In this case $i \prec_{P(n, W)} j \Longleftrightarrow X_{i} \prec X_{j}$. In other words, $P(n, W)$ then is (apart from the labelling) just the subset $\left\{X_{1}, \ldots, X_{n}\right\}$ of $\mathcal{S}$ with the induced order, provided $X_{1}, \ldots, X_{n}$ are distinct (or we regard $\left\{X_{1}, \ldots, X_{n}\right\}$ as a multiset). In this case we use also the notation $P(n, \mathcal{S})$.

One of the main results in [11] is the following representation theorem, parallel to the result for graph limits by Lovász and Szegedy [13].

Theorem 2.2. Every kernel $W$ on an ordered probability space $(\mathcal{S}, \mathcal{F}, \mu, \prec)$ defines a poset limit $\Pi_{W} \in \mathcal{P}_{\infty}$ such that the following hold.

(i) $P(n, W) \stackrel{\text { a.s. }}{\longrightarrow} \Pi_{W}$ as $n \rightarrow \infty$.

(ii) For every poset $Q \in \mathcal{P}$,

$$
t\left(Q, \Pi_{W}\right)=t(Q, W):=\int_{\mathcal{S}^{|Q|}} \prod_{i j: i<_{Q} j} W\left(x_{i}, x_{j}\right) \mathrm{d} \mu\left(x_{1}\right) \ldots \mathrm{d} \mu\left(x_{|Q|}\right) .
$$

Moreover, every poset limit $\Pi \in \mathcal{P}_{\infty}$ can be represented in this way, i.e., $\Pi=\Pi_{W}$ for some kernel $W$ on an ordered probability space $(\mathcal{S}, \mathcal{F}, \mu, \prec)$.

We also use the notation $\Pi_{W, \mu}$ or $\Pi_{\mu}$ for $\Pi_{W}$. If $W$ is as in Example 2.1, we also write $\Pi_{\mathcal{S}}$.

Unfortunately, the ordered probability space and the kernel $W$ in Theorem 2.2 are not unique (just as in the corresponding representation of graph limits); see further $[2,11]$. Nevertheless, if $W$ and $W^{\prime}$ are kernels on ordered 
probability spaces both representing the same poset limit $\Pi \in \mathcal{P}_{\infty}$, then the random posets $P(n, W)$ and $P\left(n, W^{\prime}\right)$ have the same distribution, for any $n \in \mathbb{N} \cup\{\infty\}$. We may consequently define the random poset $P(n, \Pi)$ for a poset limit $\Pi$ such that $P(n, \Pi) \stackrel{\mathrm{d}}{=} P(n, W)$ for any kernel $W$ such that $\Pi_{W}=\Pi$.

It follows easily that

$$
\mathbb{P}(P(n, \Pi)=Q)=t_{\text {ind }}(Q, \Pi),
$$

for every (labelled) poset $Q$ on $[n]$, and that the infinite random poset $P(\infty, \Pi)$ characterizes the poset limit $\Pi: P(\infty, \Pi) \stackrel{\mathrm{d}}{=} P\left(\infty, \Pi^{\prime}\right) \Longleftrightarrow \Pi=\Pi^{\prime}$ [11, Theorem 1.16].

If $\mathcal{S}$ is a measurable space, we let $\mathrm{P}(\mathcal{S})$ denote the space of probability measures on $\mathcal{S}$. We denote the Lebesgue measure on $[0,1]$ by $\lambda$.

\section{Numbers of predecessors and successors}

Given a finite poset $P$ and a point $x \in P$, define

$$
d_{-}(x):=|\{y \in P: y<x\}| \quad \text { and } \quad d_{+}(x):=|\{y \in P: y>x\}| ;
$$

these are the indegree and outdegree of $x$ in $P$ regarded as a digraph. By taking $x$ to be a uniformly random point $X$ in $P$, we obtain random variables $d_{-}(X)$ and $d_{+}(X)$; let $\nu_{ \pm}=\nu_{ \pm}(P) \in \mathrm{P}([0,1])$ be the distributions of the normalized random variables $d_{ \pm}(X) /|P|$.

For a kernel $W$ on an ordered probability space $(\mathcal{S}, \mu)$, we make the analogous definitions

$$
W_{-}(x):=\int_{\mathcal{S}} W(y, x) \mathrm{d} \mu(y) \quad \text { and } \quad W_{+}(x):=\int_{\mathcal{S}} W(x, y) \mathrm{d} \mu(y),
$$

and let $\nu_{ \pm}(W) \in \mathrm{P}([0,1])$ be the distributions of the random variables $W_{ \pm}(X)$ where $X$ is a random point with distribution $\mu$. In analogy with the degree distribution in the graph case [6, Section 4], we have the following continuity result; we equip $\mathrm{P}([0,1])$ with the usual weak topology (i.e., convergence in distribution).

Lemma 3.1. The maps $\nu_{ \pm}: \mathcal{P} \rightarrow \mathrm{P}([0,1])$ extend (uniquely) to continuous maps $\overline{\mathcal{P}} \rightarrow \mathrm{P}([0,1])$. Thus, for every poset limit $\Pi$ there exist (unique) probability distributions $\nu_{+}(\Pi)$ and $\nu_{-}(\Pi)$ on $[0,1]$ such that:

(i) If $P_{n} \rightarrow \Pi$ for a sequence of posets $P_{n}$, then $\nu_{ \pm}\left(P_{n}\right) \rightarrow \nu_{ \pm}(\Pi)$. 
(ii) The mappings $\Pi \mapsto \nu_{ \pm}(\Pi)$ are continuous on $\mathcal{P}_{\infty}$.

Moreover,

(iii) If $W$ is a kernel representing $\Pi$, then $\nu_{ \pm}(\Pi)=\nu_{ \pm}(W)$.

Proof. If $P$ is a poset and $X$ a uniform random point in $P$, then

$$
\mathbb{E}\left(d_{ \pm}(X) /|P|\right)^{k}=t\left(Q_{k}^{ \pm}, P\right),
$$

where $Q_{k}^{-}$is the poset with $k+1$ points of which one dominates everyone else and the others are incomparable, and $Q_{k}^{+}$is $Q_{k}^{-}$with the opposite order. (As digraphs, these are stars with all $k$ edges directed to [from] the centre.) Similarly, if $W$ is a kernel on $(\mathcal{S}, \mu)$ and $X$ is a random point with distribution $\mu$, then

$$
\mathbb{E} W_{ \pm}(X)^{k}=t\left(Q_{k}^{ \pm}, W\right) .
$$

Since these random variables are bounded, their distributions are determined by their moments. If $W$ and $W^{\prime}$ are two kernels representing $\Pi$, we have $t\left(Q_{k}^{ \pm}, W\right)=t\left(Q_{k}^{ \pm}, W^{\prime}\right)=t\left(Q_{k}^{ \pm}, \Pi\right)$, and thus (3.4) shows that $W_{ \pm}(X) \stackrel{\mathrm{d}}{=} W_{ \pm}^{\prime}(X)$, i.e. $\nu_{ \pm}(W)=\nu_{ \pm}\left(W^{\prime}\right)$. Consequently, we may uniquely define $\nu_{ \pm}(\Pi)=\nu_{ \pm}(W)$ when $\Pi_{W}=\Pi$, which is (iii).

(i) and (ii) follow by (3.3), (3.4) and the method of moments.

Remark 3.2. The operation $P \mapsto P^{\dagger}$ that reflects the order of $P$ extends by continuity to an involution $\overline{\mathcal{P}} \rightarrow \overline{\mathcal{P}}$. This operation interchanges $d_{-}$and $d_{+}$by (3.1), and hence it interchanges $\nu_{+}$and $\nu_{-}$on $\mathcal{P}$ and thus on $\overline{\mathcal{P}}$, i.e., $\nu_{ \pm}\left(\Pi^{\dagger}\right)=\nu_{\mp}(\Pi)$ for $\Pi \in \overline{\mathcal{P}}$.

\section{Interval orders}

A (finite) poset has an interval order if it is isomorphic to a set of intervals in $\mathbb{R}$ with $I \prec J$ if and only if $x<y$ for all $x \in I, y \in J$ (i.e., $I$ lies entirely to the left of $J$ ). See Fishburn [9] for other characterizations. We define an interval order limit to be a poset limit that is a limit of a sequence of finite posets with interval orders. We denote the set of (unlabelled) finite interval orders by $\mathcal{I O} \subset \mathcal{P}$, its closure in $\overline{\mathcal{P}}$ by $\overline{\mathcal{I O}} \subset \overline{\mathcal{P}}$ and the set of interval order limits by $\mathcal{I} \mathcal{O}_{\infty}:=\overline{\mathcal{I O}} \backslash \mathcal{I O}=\overline{\mathcal{I O}} \cap \mathcal{P}_{\infty}$.

Let $\mathcal{S}_{\mathrm{I}}:=\{[x, y]: 0 \leq x \leq y \leq 1\}$ be the set of closed intervals in $[0,1]$, with the order $I \prec J$ just defined; we identify $\mathcal{S}_{\text {I }}$ with the triangle $\{(x, y): 0 \leq x \leq y \leq 1\} \subset[0,1]^{2}$ with the partial order $\left(x_{1}, y_{1}\right) \prec\left(x_{2}, y_{2}\right)$ 
if $y_{1}<x_{2}$. (We use both interpretations of $\mathcal{S}_{\mathrm{I}}$ interchangeably below, for notational convenience.)

Any probability measure $\mu$ on $\mathcal{S}_{\text {I }}$ thus defines a distribution of random intervals. Let $W_{\mathrm{I}}$ be the kernel on $\mathcal{S}_{\mathrm{I}}$ given by $W_{\mathrm{I}}\left(\mathbf{x}_{1}, \mathbf{x}_{2}\right):=\mathbf{1}\left[\mathbf{x}_{1} \prec \mathbf{x}_{2}\right]$. Then, see Example 2.1, the random poset $P\left(n, W_{\mathrm{I}}\right)=P\left(n, W_{\mathrm{I}}, \mu\right)$ is the poset defined by $n$ random intervals (i.i.d. with distribution $\mu$ ) with the order above; thus $P\left(n, W_{\mathrm{I}}, \mu\right)$ has an interval order.

We have the following representation theorem. If $\mu$ is a measure on $\mathcal{S}_{\mathrm{I}}$, its left [right] marginal $\mu_{\mathrm{L}}\left[\mu_{\mathrm{R}}\right]$ is the distribution of the left [right] endpoint of a random interval with distribution $\mu$; thus $\mu_{\mathrm{L}}\left[\mu_{\mathrm{R}}\right]$ is the measure on $[0,1]$ obtained from $\mu$ by projecting $\mathcal{S}_{\text {I }}$ onto the first [second] coordinate.

Theorem 4.1. For every probability measure $\mu$ on $\mathcal{S}_{\mathrm{I}}$, the fixed kernel $W_{\mathrm{I}}$ on the ordered probability space $\left(\mathcal{S}_{\mathrm{I}}, \mu, \prec\right)$ defines an interval order limit $\Pi_{\mu}=$ $\Pi_{W_{\mathrm{I}}, \mu}$. Conversely, every interval order limit may be represented in this way for some (non-unique) probability measure $\mu$ on $\mathcal{S}_{\mathrm{I}}$. We may further require either that the left marginal $\mu_{\mathrm{L}}=\lambda$, or that the right marginal $\mu_{\mathrm{R}}=\lambda$.

We cannot have both $\mu_{\mathrm{L}}=\lambda$ and $\mu_{\mathrm{R}}=\lambda$ except in the trivial case when $\mu$ is concentrated on the diagonal $\{(x, x)\}$, and then the limit is the trivial poset limit $\Pi_{0}$, for which $P\left(n, \Pi_{0}\right)$ always is an anti-chain.

Proof. The poset limit $\Pi_{W_{\mathrm{I}}, \mu}$ is a.s. the limit of the interval ordered posets $P\left(n, W_{\mathrm{I}}, \mu\right)$ and is thus an interval order limit.

For the converse, we use the same arguments as for interval graph limits in [7, Section 6] (recalling that the complement of the comparability graph of an interval order is an interval graph, see Section 6). We therefore only sketch the argument and omit some details:

If $\Pi$ is an interval order limit, there are posets $P_{n}$ with interval orders and $P_{n} \rightarrow \Pi$. We may represent $P_{n}$ by intervals $I_{n i}=\left[a_{n i}, b_{n i}\right] \subseteq[0,1]$ such that the left endpoints $a_{n i}$ are evenly spaced: $a_{n i}=i /\left|P_{n}\right|$. Let $\mu_{n} \in \mathrm{P}\left(\mathcal{S}_{\mathrm{I}}\right)$ be the empirical distribution $\left|P_{n}\right|^{-1} \sum_{i} \delta_{\left(a_{n i}, b_{n i}\right)}$. By considering a subsequence, we may assume that $\mu_{n} \rightarrow \mu$ for some $\mu \in \mathrm{P}\left(\mathcal{S}_{\mathrm{I}}\right)$. It then follows that $\mu_{\mathrm{L}}=\lambda$ and $\Pi=\Pi_{\mu}$. (Note that the mapping $\mu \mapsto \Pi_{\mu}$ is not continuous, but it is continuous at every $\mu$ such that $\mu_{\mathrm{L}}$ and $\mu_{\mathrm{R}}$ do not have a common atom, and thus in particular when $\mu_{\mathrm{L}}=\lambda$ as in our case.)

Since $W_{\mathrm{I}}$ is fixed, we thus represent interval order limits by measures $\mu \in \mathrm{P}\left(\mathcal{S}_{\mathrm{I}}\right)$.

Remark 4.2. Although it is natural to represent an interval order limit by the kernel $W_{\mathrm{I}}$ on $\left(\mathcal{S}_{\mathrm{I}}, \mu\right)$ as in Theorem 4.1, it is shown in [11, Example 9.5] that any interval order limit can also be represented by a kernel on $([0,1]$, 
$\lambda,<$ ). (In this case, the space is fixed and the kernel varies. Note also that not every kernel on $[0,1]$ defines an interval order limit.)

The representation in Theorem 4.1 is not unique, but we can refine it to a unique representation. For a measure $\nu$ on $[0,1]$, define the mappings $h_{\nu}^{ \pm}:[0,1] \rightarrow[0,1]$ by

$$
\begin{aligned}
& h_{\nu}^{-}(x):=\sup \{z<x: z \in \operatorname{supp}(\nu)\}, \\
& h_{\nu}^{+}(x):=\inf \{z>x: z \in \operatorname{supp}(\nu)\},
\end{aligned}
$$

with $\sup \emptyset:=0$ and $\inf \emptyset:=1$. Write the open set $(0,1) \backslash \operatorname{supp}(\nu)$ as a union $\bigcup_{k=1}^{N}\left(a_{k}, b_{k}\right)$ of disjoint open intervals (with $0 \leq N \leq \infty$ ); then

$$
\begin{aligned}
& h_{\nu}^{-}(x)= \begin{cases}a_{k}, & \text { if } a_{k}<x \leq b_{k} \text { for some } k, \\
x, & \text { otherwise }\end{cases} \\
& h_{\nu}^{+}(x)= \begin{cases}b_{k}, & \text { if } a_{k} \leq x<b_{k} \text { for some } k, \\
x, & \text { otherwise }\end{cases}
\end{aligned}
$$

Consequently,

$$
h_{\nu}^{+} \circ h_{\nu}^{-}=h_{\nu}^{+} .
$$

We also define

$$
\bar{h}_{\nu}^{+}(x)= \begin{cases}b_{k}, & \text { if } a_{k}<x \leq b_{k} \text { for some } k, \\ x, & \text { otherwise }\end{cases}
$$

noting that $\bar{h}_{\nu}^{+}(x)=h_{\nu}^{+}(x-)$ for $x>0$ and that $\bar{h}_{\nu}^{+}(x)=h_{\nu}^{+}(x) \lambda$-a.e.

Define the mappings $H_{\nu}^{ \pm}, \bar{H}_{\nu}^{+}:[0,1]^{2} \rightarrow[0,1]^{2}$ by

$$
H_{\nu}^{ \pm}(x, y):=\left(h_{\nu}^{ \pm}(x), y\right), \quad \bar{H}_{\nu}^{+}(x, y):=\left(\bar{h}_{\nu}^{+}(x), y\right) .
$$

Further, for a measurable map $\varphi: \mathcal{S}_{1} \rightarrow \mathcal{S}_{2}$ and a measure $\mu$ on $\mathcal{S}_{1}$, let $\varphi_{*}(\mu)$ be the induced measure on $\mathcal{S}_{2}$.

Lemma 4.3. If $\mu$ is a probability measure on $\mathcal{S}_{\mathrm{I}}$, then $H_{\mu_{\mathrm{R}}}^{-}: \mathcal{S}_{\mathrm{I}} \rightarrow \mathcal{S}_{\mathrm{I}}$ and $\bar{H}_{\mu_{\mathrm{R}}}^{+}(x, y) \in \mathcal{S}_{\mathrm{I}}$ for $\mu$-a.e. $(x, y)$. Thus, $H_{\mu_{\mathrm{R}}}^{-}(\mu)$ and $\bar{H}_{\mu_{\mathrm{R}} *}^{+}(\mu)$ are probability measures on $\mathcal{S}_{\mathrm{I}}$. If $\mu_{\mathrm{L}}$ is continuous, then further $H_{\mu_{\mathrm{R}}}^{+}(x, y)=\bar{H}_{\mu_{\mathrm{R}}}^{+}(x, y) \in \mathcal{S}_{\mathrm{I}}$ for $\mu$-a.e. $(x, y)$, and thus $H_{\mu_{\mathrm{R}} *}^{+}(\mu)=\bar{H}_{\mu_{\mathrm{R}} *}^{+}(\mu) \in \mathcal{P}\left(\mathcal{S}_{\mathrm{I}}\right)$. 
Proof. The result for $H_{\mu_{\mathrm{R}}}^{-}$is obvious, since by (4.3), $0 \leq h_{\nu}^{-}(x) \leq x$.

For $\bar{H}_{\mu_{\mathrm{R}}}^{+}$, let

$$
E:=\left\{(x, y) \in \mathcal{S}_{\mathrm{I}}: \bar{H}_{\mu_{\mathrm{R}}}^{+}(x, y) \notin \mathcal{S}_{\mathrm{I}}\right\}=\left\{(x, y): 0 \leq x \leq y<\bar{h}_{\mu_{\mathrm{R}}}^{+}(x)\right\} .
$$

Write, as above, $(0,1) \backslash \operatorname{supp}\left(\mu_{\mathrm{R}}\right)=\bigcup_{k}\left(a_{k}, b_{k}\right)$ with disjoint intervals $\left(a_{k}, b_{k}\right)$. Then $x \leq y<\bar{h}_{\mu_{\mathrm{R}}}^{+}(x)$ implies, by (4.6), that $a_{k}<x \leq y<b_{k}$ for some $k$; in particular, $y \in\left(a_{k}, b_{k}\right) \subseteq(0,1) \backslash \operatorname{supp}\left(\mu_{\mathrm{R}}\right)$. Consequently, $\mu(E) \leq$ $\mu_{\mathrm{R}}\left((0,1) \backslash \operatorname{supp}\left(\mu_{\mathrm{R}}\right)\right)=0$.

Finally, if $\mu_{\mathrm{L}}$ is continuous, then $\bar{h}_{\nu}^{+}=h_{\nu}^{+} \mu_{\mathrm{L}}$-a.e., for any $\nu$, and thus $\bar{H}_{\nu}^{+}=H_{\nu}^{+} \mu$-a.e.; we choose $\nu=\mu_{\mathrm{R}}$.

Lemma 4.4. Let $\mu$ be a probability measure on $\mathcal{S}_{\mathrm{I}}$, and let $\mu_{+}=\bar{H}_{\mu_{\mathrm{R}} *}^{+}(\mu)$. Then $\Pi_{\mu_{+}}=\Pi_{\mu}$.

Note that in general, the result does not hold for $H_{\mu_{\mathrm{R}}}^{ \pm}$; this is the reason for introducing $\bar{H}_{\mu_{\mathrm{R}}}^{+}$. However, we are mainly interested in the case $\mu_{\mathrm{L}}=\lambda$, and then Lemma 4.3 shows that we can use $H_{\mu_{\mathrm{R}}}^{+}$instead of $\bar{H}_{\mu_{\mathrm{R}}}^{+}$.

Proof. Let, as above $(0,1) \backslash \operatorname{supp}\left(\mu_{\mathrm{R}}\right)=\bigcup_{k}\left(a_{k}, b_{k}\right)$ with disjoint intervals $\left(a_{k}, b_{k}\right)$.

Consider the infinite random poset $P(\infty, \mu)=P\left(\infty, W_{\mathrm{I}}, \mu\right)$. By Example 2.1, this poset is constructed by taking i.i.d. random intervals $I_{i}=\left[L_{i}, R_{i}\right]$ with distribution $\mu ; P(\infty, \mu)$ then is $\mathbb{N}$ with the order $i \prec j \Longleftrightarrow R_{i}<L_{j}$ (i.e., $\left.I_{i} \prec I_{j}\right)$. Further, $P\left(\infty, \mu_{+}\right)$is defined similarly using the intervals $I_{i}^{\prime}=\left[\bar{h}_{\mu_{\mathrm{R}}}^{+}\left(L_{i}\right), R_{i}\right]$, which have distribution $\mu_{+}$.

For every $i$ and $j, I_{i} \prec I_{j} \Longrightarrow I_{i}^{\prime} \prec I_{j}^{\prime}$, and the converse holds too except in the case $L_{j} \leq R_{i}<\bar{h}_{\mu_{\mathrm{R}}}^{+}\left(L_{j}\right)$. By (4.6), if this exceptional case holds, then, for some $k, a_{k}<L_{j} \leq R_{i}<b_{k}$; hence $R_{i} \in\left(a_{k}, b_{k}\right) \subseteq(0,1) \backslash \operatorname{supp}\left(\mu_{\mathrm{R}}\right)$. However, a.s. $R_{i} \in \operatorname{supp}\left(\mu_{\mathrm{R}}\right)$ for all $i$, and thus $I_{i} \prec I_{j} \Longleftrightarrow I_{i}^{\prime} \prec I_{j}^{\prime}$ for all $i, j$, so $P\left(\infty, \mu_{+}\right)=P(\infty, \mu)$. In other words, $P\left(\infty, \Pi_{\mu_{+}}\right) \stackrel{\mathrm{d}}{=} P\left(\infty, \Pi_{\mu}\right)$, which is equivalent to $\Pi_{\mu_{+}}=\Pi_{\mu}$, see [11].

Let $\mathrm{P}_{\mathrm{L}}\left(\mathcal{S}_{\mathrm{I}}\right):=\left\{\mu \in \mathrm{P}\left(\mathcal{S}_{\mathrm{I}}\right): \mu_{\mathrm{L}}=\lambda\right\}$. We choose to consider only representations by measures $\mu \in \mathrm{P}_{\mathrm{L}}\left(\mathcal{S}_{\mathrm{I}}\right)$ in Theorem 4.1 ; the theorem then says that $\mu \mapsto \Pi_{\mu}$ maps $\mathrm{P}_{\mathrm{L}}\left(\mathcal{S}_{\mathrm{I}}\right)$ onto $\mathcal{I} \mathcal{O}_{\infty}$. We have the following characterisation of when two measures in $\mathrm{P}_{\mathrm{L}}\left(\mathcal{S}_{\mathrm{I}}\right)$ represent the same poset limit.

Theorem 4.5. Let $\mu$ and $\mu^{\prime}$ be two measures on $\mathcal{S}_{\mathrm{I}}$ such that $\mu_{\mathrm{L}}=\mu_{\mathrm{L}}^{\prime}=\lambda$. Then the following are equivalent:

(i) $\Pi_{\mu}=\Pi_{\mu^{\prime}}$,

(ii) $H_{\mu_{\mathrm{R}}^{*}}^{-}(\mu)=H_{\mu_{R}^{\prime} *}^{-}\left(\mu^{\prime}\right)$, 
(iii) $H_{\mu_{\mathrm{R}}^{*}}^{+}(\mu)=H_{\mu_{R}^{\prime} *}^{+}\left(\mu^{\prime}\right)$,

(iv) $\mu_{\mathrm{R}}=\mu_{\mathrm{R}}^{\prime}$ and $H_{\mu_{\mathrm{R}} *}^{-}(\mu)=H_{\mu_{\mathrm{R}} *}^{-}\left(\mu^{\prime}\right)$,

(v) $\mu_{\mathrm{R}}=\mu_{\mathrm{R}}^{\prime}$ and $H_{\mu_{\mathrm{R}}^{*}}^{+*}(\mu)=H_{\mu_{\mathrm{R}} *}^{+}\left(\mu^{\prime}\right)$.

In particular, if $\mu_{\mathrm{L}}=\mu_{\mathrm{L}}^{\prime}=\lambda$ and $\operatorname{supp}\left(\mu_{\mathrm{R}}\right)=[0,1]$, then $\Pi_{\mu}=\Pi_{\mu^{\prime}} \Longleftrightarrow$ $\mu=\mu^{\prime}$.

Proof. (i) $\Longrightarrow$ (ii). Consider again the infinite random poset $P(\infty, \mu)$ constructed by i.i.d. random intervals $I_{i}=\left[L_{i}, R_{i}\right]$ with distribution $\mu$; thus $P(\infty, \mu)$ has the order $i \prec j \Longleftrightarrow R_{i}<L_{j}$. Define, for $n, i \in \mathbb{N}$,

$$
\begin{aligned}
d_{n+}(i) & :=|\{j \leq n: j \succ i\}|=\left|\left\{j \leq n: L_{j}>R_{i}\right\}\right|, \\
\tilde{d}_{n-}(i) & :=\mid\{j \leq n: \exists k \neq j \text { with } k \prec i \text { and } k \nprec j\} \mid \\
& =\left|\left\{j \leq n: \exists k \neq j: L_{j} \leq R_{k}<L_{i}\right\}\right| .
\end{aligned}
$$

By the law of large numbers, as $n \rightarrow \infty$, a.s. for every $i$,

$$
\frac{d_{n+}(i)}{n} \rightarrow \mu_{\mathrm{L}}\left(R_{i}, 1\right]=1-R_{i} .
$$

Similarly, a.s. for every $i$ and $j$,

$$
\exists k \neq i, j \text { with } L_{j} \leq R_{k}<L_{i} \Longleftrightarrow \mu_{\mathrm{R}}\left[L_{j}, L_{i}\right)>0 .
$$

Further, for all $x, y \in[0,1]$,

$$
\mu_{\mathrm{R}}(y, x)=0 \Longleftrightarrow(y, x) \cap \operatorname{supp}\left(\mu_{\mathrm{R}}\right)=\emptyset \Longleftrightarrow h_{\mu_{\mathrm{R}}}^{-}(x) \leq y .
$$

Also, since $L_{j}$ has the distribution $\mu_{\mathrm{L}}=\lambda$ which is continuous, $\mu_{\mathrm{R}}\left[L_{j}, L_{i}\right)>0 \Longleftrightarrow \mu_{\mathrm{R}}\left(L_{j}, L_{i}\right)>0$ a.s. It follows from (4.9) and (4.11)(4.12) that a.s.

$$
\tilde{d}_{n-}(i)=\left|\left\{j \leq n: \mu_{\mathrm{R}}\left(L_{j}, L_{i}\right)>0\right\}\right|=\left|\left\{j \leq n: L_{j}<h_{\mu_{\mathrm{R}}}^{-}\left(L_{i}\right)\right\}\right| .
$$

The law of large numbers now shows that, in analogy with (4.10), a.s.

$$
\frac{\tilde{d}_{n-}(i)}{n} \rightarrow \mu_{\mathrm{L}}\left[0, h_{\mu_{\mathrm{R}}}^{-}\left(L_{i}\right)\right)=h_{\mu_{\mathrm{R}}}^{-}\left(L_{i}\right) .
$$

Define

$$
Y_{i}:=\left(\limsup _{n \rightarrow \infty} \frac{\tilde{d}_{n-}(i)}{n}, 1-\limsup _{n \rightarrow \infty} \frac{d_{n+}(i)}{n}\right) \in[0,1]^{2} .
$$


We have shown in (4.10) and (4.14) that a.s. $Y_{i}=H_{\mu_{\mathrm{R}}}^{-}\left(L_{i}, R_{i}\right)$ for every $i$. By the law of large numbers again, for every continuous function $f$ on $[0,1]^{2}$, a.s.

$$
\frac{1}{n} \sum_{i=1}^{n} f\left(Y_{i}\right) \rightarrow \mathbb{E} f\left(H_{\mu_{\mathrm{R}}}^{-}\left(L_{1}, R_{1}\right)\right)=\int f \mathrm{~d} H_{\mu_{\mathrm{R}} *}^{-}(\mu) .
$$

Consequently, the measurable functional $\limsup n^{-1} \sum_{i=1}^{n} f\left(Y_{i}\right)$ of $P(n, \mu)=P\left(n, W_{\mathrm{I}}, \mu\right)$ is a.s. equal to $\int f \mathrm{~d} H_{\mu_{\mathrm{R}} *}^{-}(\mu)$.

The same applies to $\mu^{\prime}$. If (i) holds so $\Pi_{\mu}=\Pi_{\mu^{\prime}}$, then $P(n, \mu) \stackrel{\mathrm{d}}{=} P\left(n, \mu^{\prime}\right)$, and it follows that $\int f \mathrm{~d} H_{\mu_{\mathrm{R}} *}^{-}(\mu)=\int f \mathrm{~d} H_{\mu_{R}^{\prime} *}^{-}\left(\mu^{\prime}\right)$ for every continuous $f$, and thus (ii) holds.

(ii) $\Longrightarrow$ (iv). By (4.7), $H_{\mu_{\mathrm{R}}^{*}}^{-}(\mu)$ has the same right marginal as $\mu$, i.e. $\mu_{\mathrm{R}}$. Hence, (ii) implies $\mu_{\mathrm{R}}=\mu_{\mathrm{R}}^{\prime}$ and (iv) follows.

(iii) $\Longrightarrow$ (v). Similar.

(iv) $\Longrightarrow$ (ii) and (v) $\Longrightarrow$ (iii). Trivial.

(iv) $\Longrightarrow$ (v). Apply $H_{\mu_{\mathrm{R}} *}^{+}$to both sides, noting that $H_{\mu_{\mathrm{R}}}^{+} \circ H_{\mu_{\mathrm{R}}}^{-}=H_{\mu_{\mathrm{R}}}^{+}$ by (4.7) and (4.5).

$(\mathrm{v}) \Longrightarrow(\mathrm{i})$. Immediate by Lemma 4.4 , since Lemma 4.3 shows that we can use $\bar{H}_{\mu_{\mathrm{R}}}^{+}$instead of $H_{\mu_{\mathrm{R}}}^{+}$.

Finally, if $\operatorname{supp}\left(\mu_{\mathrm{R}}\right)=[0,1]$, then $h_{\mu_{\mathrm{R}}}^{-}(x)=x$ and $H_{\mu_{\mathrm{R}}}^{-}(x, y)=(x, y)$, so $H_{\mu_{\mathrm{R}} *}^{-}(\mu)=\mu$ and $H_{\mu_{\mathrm{R}} *}^{-}\left(\mu^{\prime}\right)=\mu^{\prime}$; hence (iv) implies $\mu=\mu^{\prime}$.

Let $\mathrm{P}_{\mathrm{L}}^{*}\left(\mathcal{S}_{\mathrm{I}}\right)$ be the set of $\mu \in \mathrm{P}_{\mathrm{L}}\left(\mathcal{S}_{\mathrm{I}}\right)$ such that if $(a, b)$ is an open subinterval of $(0,1)$ with $\mu_{\mathrm{R}}(a, b)=0$, then the restriction of $\mu$ to $(a, b) \times[0,1]$ is a product measure $\lambda \times \nu_{a, b}$ for some measure $\nu_{a, b}$ (necessarily supported on $[b, 1])$.

If $\mu \in \mathrm{P}_{\mathrm{L}}\left(\mathcal{S}_{\mathrm{I}}\right)$ and $(0,1) \backslash \operatorname{supp}\left(\mu_{\mathrm{R}}\right)=\bigcup_{k}\left(a_{k}, b_{k}\right)$, with $\left(a_{k}, b_{k}\right)$ disjoint, define the measures $\nu_{k}$ on $[0,1]$ by $\nu_{k}(A):=\mu\left(\left(a_{k}, b_{k}\right) \times A\right) /\left(b_{k}-a_{k}\right)$, and let $\mu^{*}$ be the measure on $[0,1]^{2}$ that equals $\mu$ on $\operatorname{supp}\left(\mu_{\mathrm{R}}\right) \times[0,1]$ and $\lambda \times \nu_{k}$ on $\left(a_{k}, b_{k}\right) \times[0,1]$.

Lemma 4.6. Let $\mu \in \mathrm{P}_{\mathrm{L}}\left(\mathcal{S}_{\mathrm{I}}\right)$. Then $\mu^{*} \in \mathrm{P}_{\mathrm{L}}^{*}\left(\mathcal{S}_{\mathrm{I}}\right)$ and $\mu \mapsto \mu^{*}$ is a projection onto $\mathrm{P}_{\mathrm{L}}^{*}\left(\mathcal{S}_{\mathrm{I}}\right)$, i.e., $\mu^{*}=\mu$ if $\mu \in \mathrm{P}_{\mathrm{L}}^{*}\left(\mathcal{S}_{\mathrm{I}}\right)$. Further, $\mu_{\mathrm{R}}^{*}=\mu_{\mathrm{R}}$ and $H_{\mu_{\mathrm{R}}}^{ \pm}(\mu)=$ $H_{\mu_{\mathrm{R}} *}^{ \pm}\left(\mu^{*}\right)$. Moreover, if $\mu, \mu^{\prime} \in \mathrm{P}_{\mathrm{L}}\left(\mathcal{S}_{\mathrm{I}}\right)$, then $H_{\mu_{\mathrm{R}} *}^{ \pm}(\mu)=H_{\mu_{\mathrm{R}}^{\prime}}^{ \pm}\left(\mu^{\prime}\right) \Longleftrightarrow \mu^{*}=$ $\left(\mu^{\prime}\right)^{*}$.

Proof. Immediate from the definitions above. (Verify $\mu_{\mathrm{R}}^{*}=\mu_{\mathrm{R}}$ first.)

This gives our desired unique representation of interval order limits. 
Theorem 4.7. The mapping $\mu \mapsto \Pi_{\mu}=\Pi_{W_{\mathrm{I}}, \mu}$ is a bijection of $\mathrm{P}_{\mathrm{L}}^{*}\left(\mathcal{S}_{\mathrm{I}}\right)$ onto the set $\mathcal{I O}_{\infty}$ of interval order limits.

Proof. If $\mu_{1}, \mu_{2} \in \mathrm{P}_{\mathrm{L}}\left(\mathcal{S}_{\mathrm{I}}\right)$, then by Theorem 4.5 and Lemma $4.6, \Pi_{\mu_{1}}=$ $\Pi_{\mu_{2}} \Longleftrightarrow \mu_{1}^{*}=\mu_{2}^{*}$. Thus, using $\mu^{* *}=\mu^{*}$, the mapping is injective on $\mathrm{P}_{\mathrm{L}}^{*}\left(\mathcal{S}_{\mathrm{I}}\right)$, and by Theorem 4.1 and Lemma 4.6 it is surjective.

Remark 4.8. This bijection is not a homeomorphism if we equip $\mathrm{P}_{\mathrm{L}}^{*}\left(\mathcal{S}_{\mathrm{I}}\right)$ with the usual subspace topology inherited from $\mathrm{P}\left(\mathcal{S}_{\mathrm{I}}\right)$; the correct topology is the quotient topology given by the quotient map $\mathrm{P}_{\mathrm{L}}\left(\mathcal{S}_{\mathrm{I}}\right) \rightarrow \mathrm{P}_{\mathrm{L}}^{*}\left(\mathcal{S}_{\mathrm{I}}\right)$ given by $\mu \mapsto \mu^{*}$.

We can also give a different type of characterization of interval order limits. Let $\mathbf{2}+\mathbf{2}$ be the poset with 4 elements $\{1,2,3,4\}$ where the only strict inequalities are $1<2$ and $3<4$ (as a digraph, the edge set $E(\mathbf{2}+\mathbf{2})=$ $\{12,34\})$. Then a poset $P$ has an interval order if and only if $P$ has no induced subposet isomorphic to $\mathbf{2}+\mathbf{2}$, i.e., $t_{\text {ind }}(\mathbf{2}+\mathbf{2}, P)=0$, see [9]. By the same argument as in the graph case [12], it is easily seen that the following holds.

Theorem 4.9. The following are equivalent, for a poset limit $\Pi$ :

(i) $\Pi$ is an interval order limit.

(ii) $t_{\text {ind }}(\mathbf{2}+\mathbf{2}, \Pi)=0$.

(iii) $P(n, \Pi)$ has a.s. an interval order for every $n$.

\section{Semiorders}

Let $\mathbf{3}+\mathbf{1}$ be the poset with 4 elements $\{1,2,3,4\}$ where $1<2<3$ but 4 is incomparable to the others. A semiorder is a partial order that does not contain any induced subposet isomorphic to $\mathbf{2}+\mathbf{2}$ or $\mathbf{3}+\mathbf{1}$, see Fishburn [9]. We define a semiorder limit as a poset limit that is the limit of a sequence of finite semiordered posets. In particular, every semiorder is an interval order and thus every semiorder limit is an interval order limit. We denote the set of (unlabelled) finite semiorders by $\mathcal{S O} \subset \mathcal{I O} \subset \mathcal{P}$, its closure in $\overline{\mathcal{P}}$ by $\overline{\mathcal{S O}} \subset$ $\overline{\mathcal{I O}} \subset \overline{\mathcal{P}}$, and the set of semiorder limits by $\mathcal{S O}_{\infty}:=\overline{\mathcal{S O}} \backslash \mathcal{S O}=\overline{\mathcal{S O}} \cap \mathcal{P}_{\infty}$; thus $\mathcal{S O}_{\infty} \subset \mathcal{I} \mathcal{O}_{\infty} \subset \mathcal{P}_{\infty}$.

The arguments in [12] show the following analogy of Theorem 4.9.

Theorem 5.1. The following are equivalent, for a poset limit $\Pi$ :

(i) $\Pi$ is a semiorder limit.

(ii) $t_{\text {ind }}(\mathbf{2}+\mathbf{2}, \Pi)=t_{\text {ind }}(\mathbf{3}+\mathbf{1}, \Pi)=0$.

(iii) $P(n, \Pi)$ is a.s. semiordered for every $n$. 
Example 5.2. Let $\Pi$ be the poset limit corresponding to the poset $\mathbf{3}+\mathbf{1}$ in the sense that $t(Q, \Pi)=t(Q, \mathbf{3}+\mathbf{1})$ for every finite poset $Q$, see [11, Example 1.12]. It follows from Theorems 4.9 and 5.1 that $\Pi \in \mathcal{I O}_{\infty}$ but $\Pi \notin \mathcal{S O}_{\infty}$. Consequently, the inclusion $\mathcal{S O}_{\infty} \subset \mathcal{I} \mathcal{O}_{\infty}$ is strict.

Example 5.3. If $\mathcal{S}$ is an ordered probability space, then the kernel $\mathbf{1}[x \prec y]$ as in Example 2.1 defines a poset limit $\Pi_{\mathcal{S}}$. Brightwell and Georgiou [5] say that $\mathcal{S}$ is an almost-semiorder if, in our notation,

$$
\mathbb{P}(P(4, \mathcal{S})=\mathbf{2}+\mathbf{2})=\mathbb{P}(P(4, \mathcal{S})=\mathbf{3}+\mathbf{1})=0 ;
$$

equivalently, see $(2.4)$, if $t_{\text {ind }}\left(\mathbf{2}+\mathbf{2}, \Pi_{\mathcal{S}}\right)=t_{\text {ind }}\left(\mathbf{3}+\mathbf{1}, \Pi_{\mathcal{S}}\right)=0$. Theorem 5.1 thus says that $\mathcal{S}$ is an almost-semiorder if and only if $\Pi_{\mathcal{S}}$ is a semiorder limit.

Since a semiorder limit is an interval order limit, it can be represented as in Theorem 4.1 or Theorem 4.7; however, only certain measures $\mu$ are possible. We will instead use a different (but related) representation. We need some preparations.

Let $\mathcal{G}$ be the set of functions $g:[0,1] \rightarrow[0,1]$ such that $g(x) \leq g(y)$ when $x \leq y$ (i.e., $g$ is weakly increasing) and $g(x) \geq x$ for all $x \in[0,1]$. If $g \in \mathcal{G}$, let $W_{g}$ be the kernel $W_{g}(x, y)=\mathbf{1}[g(x)<y]$ on $([0,1], \lambda,<)$, and let $\Pi_{g}$ be the corresponding poset limit. Note that $P\left(n, \Pi_{g}\right)=P\left(n, W_{g}\right)$ is the interval order defined by intervals $\left[X_{i}, g\left(X_{i}\right)\right]$, with $X_{1}, \ldots, X_{n}$ independent uniform random points in $[0,1]$. This is a semiorder (since $g$ is weakly increasing), and thus it follows from Theorem 5.1 that $\Pi_{g}$ is a semiorder limit.

Remark 5.4. Alternatively, we may define $\mathcal{S}_{g}$ as $[0,1]$ with the semiorder $x \prec y$ when $g(x)<y$. Thus $\mathcal{S}_{g}$ is an ordered probability space, and $\Pi_{g}$ constructed above equals $\Pi_{\mathcal{S}_{g}}$ constructed in Example 5.3.

Let $\mathcal{G}_{\text {rc }}$ be the set of right-continuous functions in $\mathcal{G}$. Note that any function $g \in \mathcal{G}$ can be modified at its jumps to become right-continuous, and that this will a.s. not change $P\left(n, W_{g}\right)$, so it will not change $\Pi_{g}$. Recall that the distribution function of a measure $\nu$ on $\mathbb{R}$ is the right-continuous function $F(t)=F_{\nu}(t):=\nu(-\infty, t]$.

Lemma 5.5. Let $g \in \mathcal{G}_{\mathrm{rc}}$, let $\nu_{ \pm}=\nu_{ \pm}\left(\Pi_{g}\right)=\nu_{ \pm}\left(W_{g}\right)$ and let $F_{ \pm}(t):=$ $\nu_{ \pm}[0, t], t \in[0,1]$, be the distribution function of $\nu_{ \pm}$. Then

$$
\begin{aligned}
& F_{-}(t)=g(t) \\
& F_{+}(t)=1-\min \{x: 1-g(x) \leq t\}=\max \{y: g(1-y) \geq 1-t\}
\end{aligned}
$$


and, symmetrically,

$$
g(x)=1-\min \left\{t: 1-F_{+}(t) \leq x\right\}=\max \left\{s: F_{+}(1-s) \geq 1-x\right\}
$$

Proof. By (3.2) and the definition of $W_{g}$,

$$
W_{g-}(x)=\int_{0}^{1} \mathbf{1}[g(y)<x] \mathrm{d} y=\sup \{y: g(y)<x\}
$$

(with $\sup \emptyset=0$ ) and thus, for $x, t \in[0,1]$,

$$
W_{g-}(x) \leq t \Longleftrightarrow(y>t \Longrightarrow g(y) \geq x) \Longleftrightarrow g(t) \geq x .
$$

Hence,

$$
F_{-}(t)=\lambda\left\{x: W_{g-}(x) \leq t\right\}=\lambda\{x: x \leq g(t)\}=g(t),
$$

showing (5.1).

Similarly, $W_{g+}(x)=1-g(x)$, so $F_{+}(t)=\lambda\{x: 1-g(x) \leq t\}$. Thus

$$
1-g(x) \leq t \Longleftrightarrow 1-F_{+}(t) \leq x, \quad t, x \in[0,1]
$$

which implies (5.2) and (5.3).

Remark 5.6. Thus, if we assume $g \in \mathcal{G}_{\mathrm{rc}}$, the relation between $g=F_{-}$and $F_{+}$is symmetric. Geometrically, we obtain the graph of $F_{-}$from the graph of $F_{+}$(or conversely) by reflection in the line $x+y=1$, adjusting the result to become right-continuous.

Theorem 5.7. If $g \in \mathcal{G}$, then $\Pi_{g}$ is a semiorder limit. Conversely, every semiorder limit equals $\Pi_{g}$ for a unique $g \in \mathcal{G}_{\mathrm{rc}}$. Thus, the mapping $g \mapsto \Pi_{g}$ is a bijection of $\mathcal{G}_{\mathrm{rc}}$ onto $\mathcal{S O}_{\infty}$.

More precisely, if $\Pi$ is a semiorder limit, then $\Pi=\Pi_{F_{-}}$where $F_{-} \in \mathcal{G}_{\mathrm{rc}}$ is the distribution function of $\nu_{-}(\Pi)$. Alternatively, if $F_{+}(t)$ is the distribution function of $\nu_{+}(\Pi)$, then $\Pi=\Pi_{g}$ where $g \in \mathcal{G}_{\mathrm{rc}}$ is given by (5.3).

Proof. We have already remarked that $\Pi_{g}$ is a semiorder limit.

Conversely, suppose that $\Pi$ is a semiorder limit. The complement of the comparability graph of a semiorder is a unit interval graph, and we argue as in [7, Section 10.4], again omitting some details: There exist semiorders $P_{n}$ with $P_{n} \rightarrow \Pi$. As in the proof of Theorem 4.1, we can represent $P_{n}$ by intervals $\left[a_{n i}, b_{n i}\right]$ with $a_{n i}=i /\left|P_{n}\right|$; further, since $P_{n}$ is a semiorder, we may assume $b_{n 1} \leq b_{n 2} \leq \ldots$ Let $\mu_{n}$ be as in the proof of Theorem 4.1, and let again $\mu \in \mathrm{P}\left(\mathcal{S}_{\mathrm{I}}\right)$ be the limit of a subsequence; thus $\Pi=\Pi_{\mu}$. Moreover, now 
the assumptions on $a_{n i}$ and $b_{n i}$ imply that if $x_{1}<x_{2}$ and $y_{1}>y_{2}$, then $\left(x_{1}, y_{1}\right)$ and $\left(x_{2}, y_{2}\right)$ cannot both belong to $\operatorname{supp}(\mu)$. Define $g$ by

$$
g(x):=\inf \{y:(z, y) \in \operatorname{supp}(\mu) \text { for some } z>x\}
$$

(with $\inf \emptyset=1$ ). Then $g \in \mathcal{G}_{\mathrm{rc}}$, and, by the property of $\operatorname{supp}(\mu)$ just shown, $\operatorname{supp}(\mu) \subseteq\{(x, y): g(x-) \leq y \leq g(x)\}$, i.e., $\operatorname{supp}(\mu)$ is a subset of the graph of $g$ with added vertical lines at the jumps. Since $\mu_{\mathrm{L}}=\lambda$, and the set of jumps of $g$ is (at most) countable, the vertical lines have measure 0 and can be ignored, and it follows that the map $\varphi:[0,1] \rightarrow \mathcal{S}_{\text {I }}$ given by $\varphi(x):=(x, g(x))$ is measure preserving $([0,1], \lambda) \rightarrow\left(\mathcal{S}_{\mathrm{I}}, \mu\right)$. (It also follows that $\operatorname{supp}(\mu)=\overline{\{(x, g(x)\}}$.) Consequently, $\Pi$, which equals $\Pi_{\mu}$ and thus can be represented by the kernel $W_{\text {I }}$ on $\left(\mathcal{S}_{\mathrm{I}}, \mu\right)$, can also be represented by the pullback $W_{\mathrm{I}}^{\varphi}$ on $([0,1], \lambda)$; moreover, it is immediately seen that $W_{\mathrm{I}}^{\varphi}=W_{g}$. Thus $\Pi$ is represented by $W_{g}$, so $\Pi=\Pi_{g}$. This shows that the mapping $g \mapsto \Pi_{g}$ is onto $\mathcal{S O}_{\infty}$.

If $\Pi=\Pi_{g}$ and $F_{-}$is the distribution function of $\nu_{-}(\Pi)$, then $g=F_{-}$ by Lemma 5.5, which shows that $g \mapsto \Pi_{g}$ is injective, and thus a bijection $\mathcal{G}_{\mathrm{rc}} \rightarrow \mathcal{S O}_{\infty}$.

The final claims follow by Lemma 5.5 .

Corollary 5.8. The mapping $\Pi \mapsto \nu_{-}(\Pi)$ is a homeomorphism of $\mathcal{S O}_{\infty}$ onto the set

$$
\mathrm{P}_{-}([0,1]):=\{\nu \in \mathrm{P}([0,1]): \nu[0, t] \geq t \text { for } t \in[0,1]\} .
$$

The inverse mapping $\mathrm{P}_{-}([0,1]) \rightarrow \mathcal{S O}_{\infty}$ is given by $\nu \mapsto \Pi_{\nu}:=\Pi_{F_{\nu}}$.

Proof. The mapping $\nu \mapsto F_{\nu}$ is a bijection of $\mathrm{P}_{-}([0,1])$ onto $\mathcal{G}_{\mathrm{rc}}$, and thus Theorem 5.7 shows that the mapping $\nu \mapsto \Pi_{F_{\nu}}$ is a bijection with inverse $\Pi \mapsto \nu_{-}(\Pi)$. Since $\nu_{-}$is continuous by Lemma 3.1 and the spaces are compact, the mappings are homeomorphisms.

Corollary 5.9. The mapping $\Pi \mapsto \nu_{+}(\Pi)$ is a homeomorphism of $\mathcal{S O}_{\infty}$ onto the set $\mathrm{P}_{-}([0,1])$. The inverse mapping $\mathrm{P}_{-}([0,1]) \rightarrow \mathcal{S O}_{\infty}$ is given by $\nu \mapsto \Pi_{\nu}^{\dagger}$.

Proof. By Corollary 5.8 and Remark 3.2.

These corollaries are analogous to the results for threshold graph limits in [6], where the limits are characterized by their degree distributions. 
Remark 5.10. The definition (5.4) says that $P_{-}([0,1])$ is the set of all distributions on $[0,1]$ that are stochastically smaller than the uniform distribution.

Corollary 5.11. Every semiorder limit equals $\Pi_{\mathcal{S}}$ for some semiordered probability space $\mathcal{S}$.

Proof. By Theorem 5.7 and Remark 5.4.

Recall that the converse holds by Example 5.3.

We proceed to corresponding limit results.

Theorem 5.12. Let $P_{n}$ be a sequence of finite semiorders with $\left|P_{n}\right| \rightarrow \infty$. Then the following are equivalent.

(i) $P_{n}$ converges to a poset limit.

(ii) The distributions $\nu_{-}\left(P_{n}\right)$ converge.

(iii) The distributions $\nu_{+}\left(P_{n}\right)$ converge.

The poset limit in (i) is necessarily a semiorder limit.

If $\nu_{-}\left(P_{n}\right) \rightarrow \nu_{-}$, then $P_{n} \rightarrow \Pi_{g}$, where $g=F_{-}$is the distribution function of $\nu_{-}$. Thus $g(t)=\lim _{n} F_{\nu_{-}\left(P_{n}\right)}(t)$ for every continuity point $t$ of $g$.

If $\nu_{+}\left(P_{n}\right) \rightarrow \nu_{+}$, then $P_{n} \rightarrow \Pi_{g}$, where $g$ is given by (5.3) where $F_{+}(t)$ is the distribution function of $\nu_{+}$. Thus $F_{+}(t)=\lim _{n} F_{\nu_{+}\left(P_{n}\right)}(t)$ for every continuity point $t$ of $F_{+}$, and we can replace $F_{+}$by $\lim \sup _{n} F_{\nu_{+}\left(P_{n}\right)}(t)$ in (5.3).

Proof. If (i) holds, then (ii) and (iii) hold by Lemma 3.1.

Assume now that (ii) holds. Thus $\nu_{-}\left(P_{n}\right) \rightarrow \nu_{-}$for some distribution $\nu_{-} \in \mathrm{P}([0,1])$. Let $F_{-}$be the distribution function of $\nu_{-}$. Suppose that $\Pi \in$ $\mathcal{P}_{\infty}$ is the limit of a subsequence of $P_{n}$. Then Lemma 3.1 and our assumption $\nu_{-}\left(P_{n}\right) \rightarrow \nu_{-}$imply that $\nu_{-}(\Pi)=\nu_{-}$. Moreover, $\Pi$ is a semiorder limit, and thus Theorem 5.7 implies that $\Pi=\Pi_{F_{-}}$.

Consequently every convergent subsequence of $P_{n}$ has the same limit $\Pi_{F_{-}}$, and (since $\overline{\mathcal{P}}$ is compact) this implies that the full sequence $P_{n}$ converges to $\Pi_{F_{-}}$. Hence (ii) $\Longrightarrow$ (i).

(iii) $\Longrightarrow$ (i) follows by a similar argument, or from the implication (ii) $\Longrightarrow$ (i) by reversing the orders as in Remark 3.2.

The final claims follow by Lemma 3.1 and Theorem 5.7, recalling that if $\nu_{n}$ and $\nu$ are probability measures on $\mathbb{R}$ with distribution functions $F_{n}$ and $F$, then $\nu_{n} \rightarrow \nu$ if and only if $F_{n}(t) \rightarrow F(t)$ at every continuity point $t$ of $F$.

This theorem extends to random posets. 
Theorem 5.13. Let $P_{n}$ be a sequence of random finite semiorders with $\left|P_{n}\right| \stackrel{\mathrm{p}}{\longrightarrow} \infty$. Then the following are equivalent.

(i) $P_{n} \stackrel{\mathrm{d}}{\longrightarrow} \Pi$ for some random poset limit $\Pi$.

(ii) $\nu_{-}\left(P_{n}\right) \stackrel{\mathrm{d}}{\longrightarrow} \nu_{-}$, for some random $\nu_{-} \in \mathrm{P}([0,1])$.

(iii) $\nu_{+}\left(P_{n}\right) \stackrel{\mathrm{d}}{\longrightarrow} \nu_{+}$, for some random $\nu_{+} \in \mathrm{P}([0,1])$.

If these hold, then $\nu_{ \pm} \stackrel{\mathrm{d}}{=} \nu_{ \pm}(\Pi)$ and $\Pi \stackrel{\mathrm{d}}{=} \Pi_{\nu_{-}}$; in particular, $\Pi$ is a.s. a semiorder limit.

As a special case, the result holds with non-random $\Pi, \nu_{-}, \nu_{+}$and $\stackrel{\mathrm{d}}{\longrightarrow}$ replaced by $\stackrel{\mathrm{p}}{\longrightarrow}$.

Proof. (i) $\Longrightarrow$ (ii), (iii). By Lemma 3.1, which also shows $\nu_{ \pm} \stackrel{\mathrm{d}}{=} \nu_{ \pm}(\Pi)$.

(ii) $\Longrightarrow$ (i). Since $\overline{\mathcal{P}}$ is a compact metric space, the space $\mathrm{P}(\overline{\mathcal{P}})$ of distributions on $\overline{\mathcal{P}}$ is compact (see e.g. [1]); thus we can select a subsequence along which $P_{n}$ converges in distribution: $P_{n} \stackrel{\mathrm{d}}{\longrightarrow} \Pi$ for some random $\Pi \in \overline{\mathcal{P}}$. It follows that a.s. $\Pi \in \mathcal{P}_{\infty}$. Moreover, if $Q=\mathbf{2}+\mathbf{2}$ or $\mathbf{3}+\mathbf{1}$, then, along the subsequence, $0=t_{\text {ind }}\left(Q, P_{n}\right) \stackrel{\mathrm{d}}{\longrightarrow} t_{\text {ind }}(Q, \Pi)$ so $t_{\text {ind }}(Q, \Pi)=0$ a.s.; hence $\Pi \in \mathcal{S O}_{\infty}$ a.s. by Theorem 5.1. Furthermore, it follows from Lemma 3.1 that, still along the subsequence, $\nu_{-}\left(P_{n}\right) \stackrel{\mathrm{d}}{\longrightarrow} \nu_{-}(\Pi)$; thus $\nu_{-}(\Pi) \stackrel{\mathrm{d}}{=} \nu_{-}$.

If $\Pi^{\prime}$ is the limit in distribution of $P_{n}$ along some other subsequence, we thus have $\nu_{-}(\Pi) \stackrel{\mathrm{d}}{=} \nu_{-}\left(\Pi^{\prime}\right)$. Since $\nu_{-}$is a homeomorphism by Corollary 5.8 , it follows that $\Pi \stackrel{\mathrm{d}}{=} \Pi^{\prime}$. Hence every convergent subsequence of the distributions $\mathcal{L}\left(P_{n}\right)$ has the same limit, and it follows (using compactness again) that the full sequence converges, i.e., (i) holds.

(iii) $\Longrightarrow$ (i). By Remark 3.2.

Finally, Corollary 5.8 yields $\Pi=\Pi_{\nu_{-}(\Pi)} \stackrel{\mathrm{d}}{=} \Pi_{\nu_{-}}$.

Example 5.14. The random graph order $G(n, p)$ is obtained by regarding $G(n, p)$ as a random directed graph, with all edges directed $i \rightarrow j$ when $i<j$, and taking the transitive closure, see e.g. [14].

These random orders are (typically) not semiorders, but Brightwell and Georgiou [5] have shown that their limits are semiorder limits: if $n \rightarrow \infty$ and $p^{-1} \log p^{-1} / n \rightarrow c$ for some $c \in[0,1]$, then $G(n, p) \stackrel{\mathrm{p}}{\longrightarrow} \Pi_{c}$, where the poset limit $\Pi_{c}$ is represented by the kernel $W_{c}(x, y):=\mathbf{1}[x+c<y]$ on $([0,1], \lambda)$; thus $W_{c}=W_{g_{c}}$ for the function $g_{c}(x):=\min (x+c, 1)$ with $g_{c} \in \mathcal{G}_{\mathrm{rc}}$, and thus $\Pi_{c}=\Pi_{g_{c}} \in \mathcal{S O}_{\infty}$. (More precisely, it suffices that $\min \left(p^{-1} \log p^{-1} / n, 1\right) \rightarrow c$, and this exhausts all possible poset limits, see [5]. In [5], the limit is described as the ordered probability space $\mathcal{S}_{g_{c}}$, see Remark 5.4 ; it is there denoted $S_{c}$.) 
We have $F_{-}\left(\Pi_{c}\right)=g_{c}$ and by an easy calculation, or by Remark 5.6, $F_{+}\left(\Pi_{c}\right)=g_{c}$ too. $\nu_{-}\left(\Pi_{c}\right)=\nu_{+}\left(\Pi_{c}\right)$ is the distribution of the random variable $\max (U-c, 0)$ with $U$ uniformly distributed on $[0,1]$.

See also [11, Example 9.4], where the kernel $W_{c}$ is denoted $W_{c^{-1}}$.

Example 5.15. Brightwell and Georgiou [5] have shown, more generally, that if a sequence of classical sequential growth models has a poset limit (in probability, see [11, Remark 4.3]), then the limit is a semiorder limit. (In [5] called an almost-semiorder, see Example 5.3.) In fact, they prove that if $P_{n}$ is a random poset of order $n$ given by some classical sequential growth model (possibly different for different $n$ ), then $\mathbb{E} t_{\text {ind }}\left(\mathbf{2}+\mathbf{2}, P_{n}\right) \rightarrow 0$ and $\mathbb{E} t_{\text {ind }}\left(\mathbf{3}+\mathbf{1}, P_{n}\right) \rightarrow 0$; if further $P_{n} \stackrel{\mathrm{p}}{\longrightarrow} \Pi$, then thus $t_{\text {ind }}(\mathbf{2}+\mathbf{2}, \Pi)=$ $t_{\text {ind }}(\mathbf{3}+\mathbf{1}, \Pi)=0$ by dominated convergence and $\Pi$ is a semiorder limit by Theorem 5.1.

Although the orders $P_{n}$ are not semiorders (typically), the proof of Theorem 5.13 still holds by the result just mentioned. (It suffices that any subsequence limit is a semiorder limit.) Thus, for example, $P_{n} \stackrel{\mathrm{p}}{\longrightarrow} \Pi$ for a (non-random) semiorder limit $\Pi$ if and only if $\nu_{-}\left(P_{n}\right) \stackrel{\mathrm{p}}{\longrightarrow} \nu_{-}$for some (non-random) distribution $\nu_{-}$, and then $\Pi=\Pi_{\nu_{-}}$.

Brightwell and Georgiou [5] gave sufficient conditions for convergence, which are related to the result just mentioned but more explicit. Under these conditions, they also gave a representation of the limit as a semiordered space which they denote by $T_{r}$, where $r:[0,1] \rightarrow[0, \infty]$ is some Borel function; in our notation the limit is $\Pi_{T_{r}}$, and it is defined by the kernel $\tilde{W}_{r}(x, y):=\mathbf{1}\left[\int_{x}^{y} r(t) \mathrm{dt}>1\right]$ on $([0,1], \lambda,<)$. This is closely related to the representation given in Theorem 5.7; in fact, $\tilde{W}_{r}=W_{g}$ where $g(x):=$ $\sup \left\{y \leq 1: \int_{x}^{y} r(t) \mathrm{d} t \leq 1\right\}$ and $T_{r}=\mathcal{S}_{g}$ defined in Remark 5.4.

It is easily seen that $g(x)=1 / 2$ for $x<1 / 4, g(x)=\min (x+1 / 2,1)$ for $x \geq 1 / 4$ cannot be represented as $\tilde{W}_{r}$, so not every semiorder limit can be represented as some $T_{r}$.

\section{Relations to interval graph limits}

Given a poset $P$, let $\Psi(P)$ denote its comparability graph, i.e., the graph with vertex set $P$ and edge set $\left\{i j: i<_{P} j\right.$ or $\left.j<_{p} i\right\}$; further, let $\bar{\Psi}(P)$ denote the complement of $\Psi(P)$. Thus $\Psi$ and $\bar{\Psi}$ are maps from the set $\mathcal{P}$ of (unlabelled) finite posets to the set $\mathcal{U}$ of (unlabelled) finite graphs; the following lemma says that these maps are continuous in the topologies used for poset limits and graph limits. As in [8], let $\overline{\mathcal{U}}$ be the completion of $\mathcal{U}$ and let $\mathcal{U}_{\infty}:=\overline{\mathcal{U}} \backslash \mathcal{U}$ be the set of graph limits. 
Lemma 6.1. The maps $\Psi$ and $\bar{\Psi}$ extend to continuous maps $\overline{\mathcal{P}} \rightarrow \overline{\mathcal{U}}$, mapping the space $\mathcal{P}_{\infty}$ of posets limits into the space $\mathcal{U}_{\infty}$ of graph limits. In particular, if a sequence of posets $P_{n}$ converges, then so do the sequences $\Psi\left(P_{n}\right)$ and $\bar{\Psi}\left(P_{n}\right)$ of graphs, and if $P_{n} \rightarrow \Pi$, then $\Psi\left(P_{n}\right) \rightarrow \Psi(\Pi), \bar{\Psi}\left(P_{n}\right) \rightarrow \bar{\Psi}(\Pi)$.

Proof. If $F$ is a finite graph, then $t_{\text {ind }}(F, \Psi(P))=\sum_{i} t_{\text {ind }}\left(F_{i}, P\right)$, where the $t$ on the left-hand side is the graph version of the functional $t$, and $F_{1}, F_{2}, \ldots$ are the different digraphs obtained by directing the edges in $F$ (it suffices to consider such digraphs that are posets, since otherwise $\left.t_{\text {ind }}\left(F_{i}, P\right)=0\right)$. This shows that the map $\Psi$ is continuous, and extends to $\overline{\mathcal{P}}$, by the definition of the topologies in $\overline{\mathcal{U}}$ and $\overline{\mathcal{P}}$, see [8] and [11].

Similarly, the map $G \mapsto \bar{G}$ mapping a graph to its complement extends to a continuous map $\overline{\mathcal{U}} \rightarrow \overline{\mathcal{U}}$ since $t_{\text {ind }}(F, \bar{G})=t_{\text {ind }}(\bar{F}, G)$. Thus the composition $\bar{\Psi}$ too is continuous.

Since $|\Psi(P)|=|\bar{\Psi}(P)|=|P|$ for any finite poset $P$, it follows easily that $\Psi$ and $\bar{\Psi}$ map $\mathcal{P}_{\infty}$ into $\mathcal{U}_{\infty}$.

\subsection{Interval orders}

If $P$ is an interval order, then $\bar{\Psi}(P)$ is an interval graph. Conversely, every interval graph can be obtained in this way. Thus, $\bar{\Psi}$ maps $\mathcal{I O}$ onto the set $\mathcal{I} \mathcal{G}$ of interval graphs. Let $\overline{\mathcal{I G}}$ be the closure of $\mathcal{I} \mathcal{G}$ in $\overline{\mathcal{U}}$ and let $\mathcal{I} \mathcal{G}_{\infty}:=\overline{\mathcal{I G}} \backslash \mathcal{I} \mathcal{G}$ be the set of interval graph limits.

Theorem 6.2. $\bar{\Psi}$ maps $\overline{\mathcal{I O}}$ onto $\overline{\mathcal{I}} \overline{\mathcal{G}}$ and $\mathcal{I O}_{\infty}$ onto $\mathcal{I G}_{\infty}$. Moreover, $\bar{\Psi}^{-1}\left(\mathcal{I} \mathcal{G}_{\infty}\right)=\mathcal{I O}_{\infty}$, i.e., if $\Pi \in \overline{\mathcal{P}}$ and $\bar{\Psi}(\Pi)$ is an interval graph limit, then $\Pi$ is an interval order limit.

Proof. Since $\bar{\Psi}: \mathcal{I O} \rightarrow \mathcal{I G}$, by continuity $\bar{\Psi}: \overline{\mathcal{I O}} \rightarrow \overline{\mathcal{I G}}$. Moreover, the range is compact, since $\overline{\mathcal{I O}}$ is compact and $\bar{\Psi}$ is continuous, and also dense in $\overline{\mathcal{I} \mathcal{G}}$ since it contains $\bar{\Psi}(\mathcal{I O})=\mathcal{I} \mathcal{G}$; hence the range $\bar{\Psi}(\overline{\mathcal{I O}})=\overline{\mathcal{I} \mathcal{G}}$. Since $\bar{\Psi}(\mathcal{I O}) \subseteq \mathcal{I} \mathcal{G}$ and $\bar{\Psi}\left(\mathcal{I} \mathcal{O}_{\infty}\right) \subseteq \mathcal{I} \mathcal{G}_{\infty}$, it follows that $\bar{\Psi}\left(\mathcal{I} \mathcal{O}_{\infty}\right)=\mathcal{I G}_{\infty}$.

If $\Pi \in \overline{\mathcal{P}}$ and $\bar{\Psi}(\Pi) \in \mathcal{I G}_{\infty}$, then $\Pi \in \mathcal{P}_{\infty}$. Furthermore, as an undirected graph, $\mathbf{2}+\mathbf{2}=\overline{\mathrm{C}}_{4}$, and thus

$$
t_{\text {ind }}(\mathbf{2}+\mathbf{2}, \Pi) \leq t_{\text {ind }}\left(\overline{\mathrm{C}}_{4}, \Psi(\Pi)\right)=t_{\text {ind }}\left(\mathrm{C}_{4}, \bar{\Psi}(\Pi)\right)=0 .
$$

Hence, $\Pi \in \mathcal{I} \mathcal{O}_{\infty}$ by Theorem 4.9 .

Using the representations of interval order limits and interval graph limits by measures $\mu \in \mathrm{P}\left(\mathcal{S}_{\mathrm{I}}\right)$ in Theorem 4.1 and [7], the map $\bar{\Psi}: \mathcal{I O}_{\infty} \rightarrow \mathcal{I} \mathcal{G}_{\infty}$ is given by $\bar{\Psi}\left(\Pi_{\mu}\right)=\Gamma_{\mu}$, since $\bar{\Psi}\left(P\left(n, \Pi_{\mu}\right)\right)=G\left(n, \Gamma_{\mu}\right)$ as is seen from the definitions of the random posets $P\left(n, \Pi_{\mu}\right)$ and the random graphs $G\left(n, \Gamma_{\mu}\right)$. 
The surjection $\bar{\Psi}: \mathcal{I O} \rightarrow \mathcal{I G}$ is not a bijection. If $G$ is a labelled interval graph, and $G$ is not complete, then there are always at least two interval orders on the vertex set that yield the graph $G$, since we may reverse any such order; these orders are sometimes, but not always equivalent as unlabelled posets. (For example, if $G$ is an empty graph on $[n]$, then any total order of $[n]$ will do, but they all yield the same unlabelled poset.) A simple example of non-uniqueness is by taking $G$ to be the disjoint sum $\mathrm{K}_{1}+\mathrm{K}_{2}+\mathrm{K}_{3}$; then $\bar{\Psi}^{-1}(G)$ consists of the 6 (weak) orders obtained by taking the three vertex sets of the subgraphs $\mathrm{K}_{1}, \mathrm{~K}_{2}, \mathrm{~K}_{3}$ in any order, letting the vertices inside each set be incomparable to each other. See further [9, Section 3.6] (labelled case) and [10] (unlabelled case); these references contain among other results characterizations of interval graphs $G$ such that $\bar{\Psi}^{-1}(G)$ contains only one poset, or two poset with opposite orders.

Problem 6.3. Describe $\bar{\Psi}^{-1}(\Gamma)$ for a general interval graph limit $\Gamma$. In particular, characterize the interval graph limits $\Gamma$ such that $\bar{\Psi}^{-1}(\Gamma)$ consists of a single poset limit, or two poset limits related by reflection.

It seems likely that a solution to this problem could be combined with the unique representation in Theorem 4.7 to yield a unique representation of interval graph limits by some class of kernels.

\subsection{Semiorders}

$P$ is a semiorder if and only if $\bar{\Psi}(P)$ is a unit interval graph (a.k.a. indifference graph); moreover, every unit interval graph is $\bar{\Psi}(P)$ for some semiorder $P$ [9, Theorem 3.2]. Thus, $\bar{\Psi}$ maps $\mathcal{S O}$ onto the set $\mathcal{U I G}$ of unit interval graphs. Let $\overline{\mathcal{U I G}}$ be the closure of $\mathcal{U} \mathcal{I G}$ in $\overline{\mathcal{U}}$ and let $\mathcal{U} \mathcal{I} \mathcal{G}_{\infty}:=\overline{\mathcal{U I C}} \backslash \mathcal{U} \mathcal{I G}$ be the set of unit interval graph limits.

Theorem 6.4. $\bar{\Psi}$ maps $\overline{\mathcal{S O}}$ onto $\overline{\mathcal{U I G}}$ and $\mathcal{S O}_{\infty}$ onto $\mathcal{U} \mathcal{I} \mathcal{G}_{\infty}$. Moreover, $\bar{\Psi}^{-1}\left(\mathcal{U} \mathcal{I} \mathcal{G}_{\infty}\right)=\mathcal{S O}_{\infty}$, i.e., if $\Pi \in \overline{\mathcal{P}}$ and $\bar{\Psi}(\Pi)$ is a unit interval graph limit, then $\Pi$ is a semiorder limit.

Proof. As the proof of Theorem 6.2, mutatis mutandis; now using Theorem 5.1.

Problem 6.3 seems considerably easier for unit interval graph limits in view of the results for unit interval graphs in $[9,10]$. In particular, if $G$ is a connected unit interval graph, then $\bar{\Psi}^{-1}(G)$ consists of just one or two posets $P$ and $P^{\dagger}$ (which may be the same or not, as unlabelled posets) [9, Theorem 3.10]. This leads to the following conjecture. 
Conjecture 6.5. If $\Gamma$ is a connected unit interval graph limit, then $\bar{\Psi}^{-1}(\Gamma)$ consists of either one semiorder limit $\Pi$ (with $\Pi^{\dagger}=\Pi$ ) or two semiorder limits $\Pi$ and $\Pi^{\dagger}$.

If this conjecture would be proven, it would by Theorem 5.7 lead to a result on characterization of representations of unit interval graph limits, and perhaps to a way of selecting unique or almost unique such representations.

\section{References}

[1] Billingsley, P. (1968). Convergence of Probability Measures. Wiley, New York. MR0233396

[2] Borgs, C., Chayes, J. T. and Lovász, L. (2010). Moments of two-variable functions and the uniqueness of graph limits. Geom. Funct. Anal. 19(6) 1597-1619. MR2594615

[3] Borgs, C., Chayes, J. T., Lovász, L., Sós, V. T. and Vesztergombi, K. (2008). Convergent sequences of dense graphs. I. Subgraph frequencies, metric properties and testing. Adv. Math. 219(6) 1801-1851. MR2455626

[4] Borgs, C., Chayes, J. T., Lovász, L., Sós, V. T. and Vesztergombi, K. (2007). Convergent sequences of dense graphs II: Multiway cuts and statistical physics. Preprint. http://research.microsoft.com/ borgs/

[5] Brightwell, G. and Georgiou, N. (2010). Continuum limits for classical sequential growth models. Random Structures Algorithms 36(2) 218250. MR2583061

[6] Diaconis, P., Holmes, S. and Janson, S. (2009). Threshold graph limits and random threshold graphs. Internet Mathematics 5(3) 267-318. MR2573956

[7] Diaconis, P., Holmes, S. and Janson, S. Interval graph limits. Ann. Combinatorics, to appear.

[8] Diaconis, P. and Janson, S. (2008). Graph limits and exchangeable random graphs. Rendiconti di Matematica 28 33-61. MR2463439

[9] Fishburn, P. C., (1985). Interval Orders and Interval Graphs. A Study of Partially Ordered Sets. Wiley, Chichester. MR0776781

[10] Hanlon, P. (1982). Counting interval graphs. Trans. Amer. Math. Soc. 272(2) 383-426. MR0662044 
[11] Janson, S. (2011). Poset limits and exchangeable random posets. Combinatorica 31(5) 529-563. MR2886098

[12] Janson, S. (2011). Graph limits and hereditary properties. Preprint. arXiv:1102.3571.

[13] Lovász, L. and Szegedy, B. (2006). Limits of dense graph sequences. J. Comb. Theory B 96 933-957. MR2274085

[14] Pittel, B. and Tungol, R. (2001). A phase transition phenomenon in a random directed acyclic graph. Random Struct. Alg. 18(2) 164-184. MR1809721

SVANTE JANSON

DEPARTMENT OF MATHEMATICS

UPPSALA UNIVERSITY

PO BOX 480

75106 UPPSALA

SWEDEN

E-mail address: svante.janson@math.uu.se

Received January 12, 2012 